\title{
5. Symposium über Fragen der Cyanophytensystematik in Kastanienbaum, 1969
}

\section{Verhandlungsbericht}

Von Alfons ZeHnder

Manuskript eingegangen am 29. Mai 1970

\section{Einleitung}

Am 17. Kongress der Internationalen Vereinigung für theoretische und angewandte Limnologie in Jerusalem im August 1968 beschlossen die dort anwesenden Mitglieder der Internationalen Arbeitsgemeinschaft für Cyanophytenforschung, das 5. Symposium über Fragen der Blaualgensystematik vom 23. Juli bis 1. August 1969 wiederum im Hydrobiologischen Laboratorium der Eidgenössischen Technischen Hochschule in Kastanienbaum bei Luzern abzuhalten. Erfreulicherweise meldeten sich neben Teilnehmern an früheren Symposien neue Kolleginnen und Kollegen an, und der Leiter der Arbeitstagung, Herr Professor Dr. OtTo JAAG, konnte in Kastanienbaum die folgenden Fachleute willkommen heissen:

Dr. Diedrich Backhaus, Karlsruhe

Dr. KarL Behre, Bremen-Lesum

Dr. Marianne Bosli-Pavoni, Zürich

Prof. Dr. Pierre Bourrelly, Paris

$M^{\text {me }}$ Thérèse Le Campion-Alsumard, Marseille

Prof. Dr. Richard W. Castenholz, Eugene, Oregon

Prof. Dr. Heinz Ciémençon, Lausanne

Dr. Yvonne J. Le Cosquino de Bussy, Delft

Prof. Dr. T. V. Desikachary, Madras

Dr. Elie EICHEnberGer, Zürich

Dr. Einer Fjerdingstad, Kopenhagen

Dr. Günther Friedrich, Krefeld-Hülserberg

Dr. Stjepko Golubić, Wayne, N. J. 
Dr. Alida Jaarsveld, Amsterdam

Dr. Edith KanN, Wien

Dr. JiRI KomareK, Trěbon

Dr. Dieter Mollenhauer, Bieber-Spessart

Prof. Dr. Karol Starmach, Krakau

Prof. Dr. Eugen A. Thomas, Zürich

Dr. Brian Whitton, Durham

Dr. Alfons Zehnder, Neuenhof AG

Für die Organisation sorgten Mitarbeiter von Professor Dr. JAAG, nämlich Dr. Hernz AmbüHL, Chef der Limnologischen Abteilung der EAWAG, Frau Dr. MARIanne Bosli-Pavoni, Fräulein Beatrix Egli sowie der Berichterstatter.

Auf Grund eines am 4. Symposium 1966 gefassten Beschlusses stand als Hauptthema im Zentrum des Programms: "Die taxonomische Begrenzung des Formenkreises Oscillatoria - Phormidium - Lyngbya sowie nahestehender Gattungen wie Symploca, Hydrocoleum.»

Wie an früheren Symposien sollte das Hauptthema aber eher Richtschnur als conditio sine qua non sein. So führten denn auch diesmal mehrere der zwanzig Kurzreferate in andere Bereiche der Systematik und Ökologie der Blaualgen. Die meisten Mitteilungen bildeten Ausgangspunkte für Diskussionen und die bewusst ins Zentrum gestellte Arbeit am Mikroskop. Neun Referate wurden in erweiterter Form als druckfertige Manuskripte eingereicht und finden sich im Anschluss an den vorliegenden Bericht publiziert (Seite $490 \mathrm{ff}$.), wobei zwei Arbeiten von Autoren stammen, die nicht selber am Symposium teilnehmen konnten (A. Watanabe; F. Marty und F. Busson). Im folgenden werden die an den Sitzungen gehaltenen Vorträge mit den zugehörigen Diskussionen kurz zusammengefasst. Gleichzeitig sei nochmals betont, dass die Arbeit am Mikroskop bewusst im Zentrum der Tagung stand. Die dabei gemeinsam erarbeiteten Erkenntnisse sollen hier nicht im einzelnen dargelegt werden. So wie die mikroskopische Arbeit in früheren Symposien zu Arbeiten anregte, deren Ergebnisse hier in Form von Referaten dargestellt werden, so werden auch die diesmaligen mikroskopischen Untersuchungen - so hofft der Berichterstatter - zu neuen Arbeiten anregen.

\section{Grundsatzreferate zur Blaualgentaxonomie}

Desikachary, T.V.: Taxonomy of Blue-Green Algae: Problems and Prospects (Seite 490)

Ein Blick in die Geschichte der Blaualgensystematik zeigt sofort ihre Schwierigkeiten und Probleme. Um die Lösung nomenklatorischer Fragen machten sich DroueT und DaILY verdient; die taxonomischen Probleme wurden mit ihren Arbeiten aber nicht gelöst. So stellt etwa die Geschichte der für physiologische Arbeiten oft benützten Anacystis nidulans geradezu eine Tragödie dar. Entscheidende Fortschritte in der Blaualgensystematik sind erst zu erwarten, wenn die Untersuchung von in der Natur gesammelten Materialien kombiniert wird mit Beobachtungen an kultiviertem Material vom gleichen Standort. Für taxonomische Arbeiten sind einwandfreie Klonkulturen, die aber nicht bakterienfrei sein müssen, notwendig. Dabei sollten neu zur Untersuchung gelangende Formen parallel mit bereits bekannten, nahe verwandten 
Formen kultiviert werden. Nur so lässt sich sicher entscheiden, ob tatsächlich ein neues Taxon vorliegt. Natur- und Kulturmaterialien müssen statistisch bearbeitet werden. Die figürliche Darstellung von Variationspolygonen (z.B. der Trichombreiten) gibt bessere und anschaulichere Aufschlïsse als die blosse Angabe eines Mittelwertes und seiner Standardabweichung. Noch besser als mit dem Variationspolygon kann die Variabilität freilich mittels der «Cumulative Frequency Curve» dargestellt werden. In manchen Fällen können physiologische Befunde zur Klärung taxonomischer Fragen beitragen. Beim Einbezug der Physiologie stellt sich aber sofort die Frage nach einer geeigneten Standardnährlösung für die Kulturen.

Diskussion. JAAG verweist in seinen ergänzenden Ausführungen auf drei grundlegende Mängel in fast der gesamten Literatur über Blaualgensystematik: 1. Die Masse werden oft falsch angegeben, weil die von älteren Autoren wie DE CANDOLLE verwendete Längeneinheit "Linie» falsch in das metrische System umgerechnet wurde. Die falschen Umrechnungen gingen in die gesamte Literatur ein. 2. Die Plastizität vieler Blaualgen wird nicht oder doch zu wenig berücksichtigt. 3. Merkmale ohne taxonomischen Wert werden als bedeutsam hingestellt.

Komarek, J.: Das Studium der Blaualgenvariabilität in Kulturen: Möglichkeiten für die Taxonomie

Die Kulturmethode gibt uns zwar keinen Schlüssel für die endgültige Beantwortung schwieriger Fragen der Blaualgensystematik; sie ist jedoch ein wichtiges Hilfsmittel und erweist sich als besonders nützlich 1. zum Studium der Variabilität eines Merkmals unter dem Einfluss eines Umweltfaktors, 2. zur Feststellung, ob zwischen zwei Stämmen morphologische oder physiologische Unterschiede bestehen, 3. zum Studium der Variabilität eines Klons unter verschiedenen Umweltbedingungen. Freilich setzen sich einem ausgedehnteren Einsatz der Kulturmethode zur Lösung taxonomischer Fragen noch manche Schwierigkeiten entgegen:

1. Aus vielen Gattungen sind erst ganz vereinzelte Arten isoliert und kultiviert worden, und auch diese Kulturen entstanden grösstenteils zufällig und nicht gezielt. Es handelt sich in der Regel um Kulturen leicht wachsender Arten.

2. Sollen ähnliche Formen verglichen werden, so soliten sie parallel unter gleichen Bedingungen kultiviert werden. Dabei sind aber eventuell ihre physiologischen und ökologischen Ansprüche verschieden. Das kann dazu führen, dass zum Beispiel

Stamm 1 unter den Umweltbedingungen A die Merkmalskombination X, Stamm 2 unter den Umweltbedingungen A die Merkmalskombination $\mathrm{Y}$, Stamm 2 unter den Umweltbedingungen B die Merkmalskombination X zeigt. Sind nun die Stämme 1 und 2 als taxonomisch identisch zu betrachten?

3. Koloniebildende Formen wie Aphanizomenon flos-aquae bilden in der Kultur in der Regel keine Kolonien.

4. In Standkulturen ändern sich die Bedingungen ständig. Die Kultur unter konstanten Bedingungen - Durchflusskultur - ist aber sehr aufwendig.

Trotz diesen Schwierigkeiten muss die Kulturmethode in die taxonomischen Arbeiten miteinbezogen werden, denn wirkliche Fortschritte werden sich in der Blaualgensystematik nur durch gleichzeitiges Studium von Natur- und Kulturmaterial erzielen lassen. 
Diskussion. Sie befasst sich besonders mit der Frage, welche Merkmalsgruppen taxonomisch bedeutsam seien: Sicher die Morphologie des Individuums, sodann physiologische Unterschiede, zum Beispiel die Fähigkeit, ein bestimmtes Toxin zu bilden oder nicht zu bilden, wobei allerdings sich nur so unterscheidende Taxa nicht auf Spezies-Niveau gehoben, sondern eher als Varietäten unterschieden werden sollen. Auch Formen, die sich nur in der Gestalt ihrer Kolonien unterscheiden, sollen nicht als verschiedene Arten, sondern als «Stämme» der gleichen Art bezeichnet werden.

\section{Beiträge zur Taxonomie der Oscillatoriaceae}

Bourrelly, P.: Note sur la famille des Oscillatoriacées (Seite 519)

Die Familie der Oscillatoriaceae ist als Ganzes sehr homogen. Die Taxa können charakterisiert werden auf Grund des Aussehens der einzelnen Zellen (zum Beispiel Zellen mit oder ohne Pseudovakuolen), der Trichome (zum Beispiel Enden verjüngt oder unverjüngt) und der Lager (zum Beispiel hautartig oder samtartig). Die Erfassung und Darstellung der spezifischen Thallusmerkmale erweist sich dabei als besonders schwierig. Lyngbya, Phormidium und Symploca sollten eigentlich zu einer einzigen Gattung vereinigt werden. Schwierige Probleme stellen zum Beispiel Pseudoanabaena (treten Akineten auf oder nicht?), Isocystis (Typus nicht mehr auffindbar), Borzia (Typusmaterial enthält wohl nur keimende Hormogonien).

Diskussion. Die Bedeutung einiger Merkmale für die Systematik der Familie wird erörtert, so die Bedeutung der Scheide: In Kulturen bilden die Lyngbyaarten offenbar immer Scheiden, während einige Phormidien manchmal Scheiden bilden, manchmal nicht. Für die systematische Charakterisierung müssen in Zukunft auch Eigenschaften herangezogen werden, deren Untersuchung bisher vernachlässigt wurde, zum Beispiel die Drehrichtung der Fäden, gibt es doch konstant linksdrehende und konstant rechtsdrehende Oszillatoriazeen.

KAnn, E.: Systematische und ökologische Bemerkungen zu einigen Arten von Phormidium (Seite 495)

In stehenden und fliessenden Gewässern kommen sehr häufig dunkelblaugrüne Lager von Phormidium vor, aus einer oder mehreren Arten bestehend. Da die Phormidien wenige morphologische Merkmale aufweisen, sind sie schwer zu bestimmen. Um etwas Klarheit in die Systematik zu bringen, wurden die Merkmale einiger häufiger Arten miteinander verglichen. Die berücksichtigten Arten sind: Phormidium autumnale, Ph. uncinatum, Ph. favosum, Ph. subfuscum, Ph. Retzii, Ph. incrustatum, Ph. umbilicatum, Ph. toficola, Ph. Corium, Ph. inundatum, Ph. foveolarum. Nach GeITLER (1932) sind folgende Merkmale «wesentlich»: Grösse, Gestalt des Trichomendes, Endzelle. Hingegen sind Scheidenbildung, Drehung «unsichere Merkmale». Bei Berücksichtigung nur der wesentlichen Merkmale (Trichome 3-9 $\mu$ breit, am Ende gerade oder gebogen, Endzelle kopfig, Kalyptra halbkugelig-kegelig) ergibt sich unter den Arten Ph. autumnale, uncinatum, favosum eine derart grosse Übereinstimmung - auch in ökologischer Beziehung -, dass diese Arten identisch zu sein scheinen und für sie die Bezeichnung Ph. autumnale gelten müsste. Auch ein Vergleich der in der Literatur vorhandenen Abbildungen bestätigt im grossen ganzen diesen Eindruck. - Die Art Phormidium subfuscum ist den vorigen ähnlich, doch lässt sie sich durch ihre geringe 
Zellänge, das gerade Ende und die kegelige Endzelle deutlich von ihnen abgrenzen und ist deshalb als eigene Art aufrechtzuerhalten. Wenn auch ungefähr in die gleiche Grössenordnung gehörend, unterscheidet sich $P h$. Retzii doch sehr deutlich durch die charakteristisch abgestumpfte Endzelle, wodurch die Bestimmung relativ leicht ist. Unklarheit besteht bei jenen Phormidium-Arten, deren Lager mit Kalk inkrustiert sind: Ph. incrustatum, umbilicatum, toficola. Es ist nach Beobachtungen bei anderen Gattungen zweifelhaft, ob dieses Bestimmungsmerkmal seine Gültigkeit hat, wenngleich diese Arten nebst anderen Cyanophyceen den Hauptteil der Algen gewisser Kalkinkrustationen ausmachen. Die drei Arten zeigen in der Trichombreite, dem kurz verjüngten Ende ohne Kalyptra und der steinernen Lagerbildung soviel Übereinstimmung, dass sie wohl identisch sind und die Bezeichnung Ph. incrustatum tragen müssen. - Phormidium Corium ist den eben genannten Arten ähnlich, kommt aber nicht in Inkrustationen vor und ist deutlich an ihren viel längeren Zellen erkennbar. Sie kann daher als eigene Art gelten. Mit ihr stimmt $P h$. inundatum so weitgehend überein, dass auch hier Identität vermutet werden kann, obwohl in der Literatur bisher nicht darauf verwiesen wurde. - An reinen und an verunreinigten Stellen der Gewässer ist $P h$. foveolarum sehr häufig. Wegen ihrer Feinheit und Merkmalsarmut ist sie schwer zu bestimmen und leicht mit ähnlichen Arten wie Homoeothrix varians oder Lyngbya Kützingii zu verwechseln. Ein charakteristisches Merkmal jedoch - getrennte Zellen - kommt in Abbildungen neuerer Arbeiten deutlich zum Ausdruck und ist bisher zu wenig beachtet worden, obwohl es die Bestimmung erleichtert.

Diskussion. Die Diskussionen zu diesem und den vier folgenden Referaten finden sich nach dem Referat von Thомas, Seite 486, zusammengefasst.

Komarek, J.: Über die Variabilität bei Phormidium autumnale (Ag.) Gom. und Ph. uncinatum (Ag.) Gom. in Kultur (Seite 495)

Der Autor untersuchte den Einfluss verschiedener Temperaturen, Belichtungsintensitäten und des Substrates (fest oder flüssig) auf vierzehn Stämme der Phormidium-autumnale-Gruppe, welche in der Algothek in Trěbon gehalten werden. Auf Grund ihres Verhaltens in den Kulturen lassen sich die Stämme in drei Gruppen gliedern:

1. Gruppe: Ein Stamm aus Kuba. Entspricht genau dem Phormidium ambiguum Gom. Kalyptra nur bei hohen Temperaturen ausgebildet.

2. Gruppe: Zwei Stämme. Sie entsprechen $P h$. subfuscum und $P h$. uncinatum, die miteinander zu vereinigen und als $P h$. subfuscum zu bezeichnen sind.

3. Gruppe: Elf Stämme. Sie unterscheiden sich in Einzelheiten, zeigen aber fliessende Úbergänge. Sie entsprechen $P h$. autumnale, $P h$. setchellianum, $P h$. favosum und $P h$. calidum, die zu vereinigen und als $P h$. autumnale zu bezeichnen sind.

Der wünschbare unmittelbare Vergleich der Kulturen mit dem Naturmaterial, aus dem die Kulturen gewonnen worden waren, konnte nicht durchgeführt werden. Form und Farben der Lager hängen vom physiologischen Alter der Kulturen ab. Günstige Wachstumsbedingungen erzeugen schwarzgrüne, ungünstige hellgrüne bis gelbe Lager. Die Fähigkeit, eine Kalyptra zu bilden, wurde als gutes Artmerkmal erkannt. Die effektive Bildung einer Kalyptra bei einem bestimmten Stamm hängt von den Kulturbedingungen ab. Granulierung des Zellinhaltes scheint ausschliesslich von Kulturbedingungen abzuhängen; sie tritt ein, wenn die Kulturen von der exponentiellen in die stationäre Wachstumsphase übergehen. 
EGLI, B.: Trichombreiten von Phormidium autumnale in Kulturen auf festen und flüssigen Nährböden (Wird später in erweiterter Form publiziert)

Ein Stamm aus der Phormidium-autumnale-Gruppe, der aus einem Material vom Grunde des Hochrheines isoliert worden war, wurde während fünf Jahren parallel auf festen Agarnährböden und in Nährlösung in Standkulturen gezogen. Überimpfungen auf frische Substrate wurden in gleichen Abständen vorgenommen. Periodisch wurden die Trichombreiten gemessen und statistisch bearbeitet. Sie blieben während der fünfjährigen Beobachtungsperiode praktisch konstant.

Bosli-Pavoni, M.: Über die Verteilung von Phormidien in Modellfliessgewässern

Die Verteilung der Phormidien in den Fliesswasserrinnen der Versuchsstation Tüffenwies der EAWAG wurde untersucht, wobei besonders die Phormidium-autumnale-Gruppe berücksichtigt wurde. Es konnten violettgrüne und blaugrüne Formen von $P h$. autumnale festgestellt werden, wobei in den ersteren Phycoerythrin nachweisbar war, das den letzteren fehlte. Beobachtungen und Versuche zeigten, dass die Fähigkeit, Phycoerythrin zu bilden bzw. nicht zu bilden, genetisch fixiert ist. Die Arbeit an den Modellfliessgewässern zeigte einmal mehr die Unberechenbarkeit des Auftretens von Blaualgen. Diese vorläufige Unberechenbarkeit des Auftretens und Verschwindens ist eine auch bei planktischen Blaualgen wie Oscillatoria rubescens oder Aphanizomenon flos-aquae immer wieder überraschende Erscheinung.

Thomas, E.A.: Beobachtungen über das Wandern von Phormidium autumnale und Oscillatoria princeps auf Agar (Seite 523)

Es wurde der Einfluss des Lichtes auf die Ausbildung der Lager der beiden Algen untersucht: Das spiralige Auswachsen der Fäden auf festen Agarsubstraten von der Impfstelle aus ist eine Folge der Drehung der Fäden um die eigene Längsachse. Die Drehrichtung scheint genetisch fixiert zu sein. Sie sollte bei der Beschreibung neuer Taxa mitberücksichtigt werden.

Diskussion. In der gemeinsamen Aussprache über die Referate von KanN (Seite 495), Komarek (Seite 495), Egli (Seite 486), Bosli-Pavoni (Seite 486) und Thomas (Seite 523) wurde die taxonomische Bedeutung einiger Merkmale, zum Beispiel der Kalkinkrustation bei verschiedenen Arten, erörtert. So inkrustiert Rivularia Biasolettiana meist Kalk, ist aber im Lago Maggiore kalkfrei. Gongrosira incrustans zeigt an Bachstellen ohne freies Kohlendioxid im Wasser Kalkinkrustation; an Stellen des gleichen Baches, an denen das Wasser freies Kohlendioxid enthält, ist sie aber nicht inkrustiert. Diese und andere Beispiele zeigen, dass in der Taxonomie zwei grundsätzlich verschiedene Gruppen von Merkmalen unterschieden werden müssen: 1. morphologische Eigenschaften, die immer vorhanden sind, wie bestimmte Zellgrössen, Trichombreiten usw., und 2. Fähigkeiten, etwas zu tun oder nicht zu tun, zum Beispiel die Fähigkeit, unter gewissen Umweltbedingungen Kalk zu inkrustieren oder Phycoerythrin zu bilden. Die solchen Fähigkeiten entspringenden morphologischen Strukturen sind bei einem Taxon je nach den Entwicklungsbedingungen einmal vorhanden, ein anderes Mal nicht. Das Fehlen kann in diesen Fällen selbstverständlich kein neues Taxon begründen. Es wird dabei deutlich, dass jedes Merkmal einen genetischen und einen ökologischen Aspekt hat. - Die Bedeutung einer guten Formenkenntnis für den Ökologen wurde schliesslich besonders betont. 


\section{Mitteilungen aus verschiedenen Gebieten der Blaualgensystematik}

Mollenhauer, D.: Die sogenannte Moorschnecke (Seite 532)

In Hochmooren wird hin und wieder ein eigenartiges Gebilde gefunden, dem F. STEINECKE bei seinen Untersuchungen des Zehlau-Hochmoores in Ostpreussen den Namen «Moorschnecke» gegeben hat. Schon vorher und bis heute immer wieder finden sich Fundmeldungen der Moorschnecke in der phykologischen, vor allem der floristischen Literatur. GESSNER nennt die "Moorschnecke» in seiner 》 Hydrobotanik II" (Seite 268) eine "echte Hochmoorblaualge». Seit ihrer ersten Beschreibung bei SCHLENKER 1908 wird bis heute - zuletzt bei HüLsBRUCH 1967 - die Frage diskutiert, wo die «Moorschnecke» einzuordnen sei, ob bei den Blaualgen (Familie Microchaetaceae) oder bei den Pilzen. Der Kulturversuch, ergänzt um mikrochemische Befunde, klärt dieses Problem. Es handelt sich um didymospore, helicospore DeuteromycetenKonidien. Der sie erzeugende Pilz gehört in die Gattung Helicoon Morgan aus der Familie Dematiaceae innerhalb der Moliniales. Er konnte im Labor kultiviert und zur Sporulation gebracht werden.

Diskussion. Bei genauer mikroskopischer Beobachtung lässt sich schon aus der Struktur der Zellwände auf die Pilznatur der «Moorschnecke» schliessen.

Schwabe, G. H., und Behre, K.: Zur Neubesiedlung der Vulkaninsel Surtsey/Island (Die Arbeit wird an anderer Stelle ausführlich publiziert)

Schon im Juli 1968, also kaum viereinhalb Jahre nach dem ersten Auftauchen der Insel im Januar 1964 oder drei bzw. ein Jahr nach dem Ende der Eruptionen konnte in den Dampfzonen von zwei Kraterräumen eine artenreiche Algenflora festgestellt werden. Identifiziert wurden 8 Cyanophyten-, 13 Flagellaten-, 9 Chlorophyten- und Xanthophyten-, 74 Diatomeen-Taxa. Auffallend ist das Überwiegen kleiner Formen. Als Hauptverfrachter der Süsswassermikroorganismen auf die neue Insel kommen Wind und - in geringerem Masse - Vögel in Frage.

ZEHNDER, A.: Blaualgen aus Nepal (Die Arbeit wird später in erweiterter Form publiziert)

In Nepal gesammelte Scytonemen und ein Porphyrosiphon wurden in fixiertem Zustand sowie als Klonkulturen demonstriert. Die statistische Bearbeitung von drei Scytonema-Naturmaterialien und von aus ihnen gewonnenen Klonkulturen zeigte, dass die Trichombreiten in den Kulturen eindeutig über jenen der Naturmaterialien lagen. Die Erscheinung wird auf das höhere Nährstoffangebot in den Kulturen zurückgeführt.

ZIMMERMANN, U.: Ökologische und physiologische Untersuchungen an Oscillatoria rubescens D.C. (Die Arbeit ist in Vol. 31, Fasc. 1 [1969] dieser Zeitschrift ausführlich publiziert)

Beobachtungen in der Natur und experimentelle Untersuchungen mit einem bakterienfreien Klon zeigten, dass Oscillatoria rubescens ein eurythermer, oligophoter Organismus ist.

Diskussion. Es wurde insbesondere darauf hingewiesen, dass ein Vergleich der Lichtverhältnisse am natürlichen Standort mit jenen im Laboratoriumsversuch äusserst schwierig ist. 
Castenholz, R.W.: Laboratory Culture of Thermophilic Cyanophytes (Seite 538)

An der University of Oregon wird eine Kultursammlung thermophiler Blaualgen aufgebaut. Sie umfasst momentan rund 70 Stämme. Die meisten werden bei $45^{\circ} \mathrm{C}$ in einem anorganischen Medium gehalten. Einige Mastigocladusstämme werden bei $60^{\circ} \mathrm{C}$, ein Synechococcus ausserdem bei $65^{\circ} \mathrm{C}$ und bei $70{ }^{\circ} \mathrm{C}$ kultiviert. Die meisten Stämme erweisen sich bisher morphologisch und in ihren Temperaturansprüchen als stabil.

Diskussion. Besonderes Interesse galt den Beziehungen zwischen Blaualgen und Bakterien in Warmkulturen; dabei zeigte sich rasch, dass darüber noch sehr wenig bekannt ist.

Whitton, B.: Range of Blue-Green Algal Forms in one Defined Geographical Area (Aldabra, a Coral Island, $9^{\circ} \mathrm{S}, 46^{\circ} \mathrm{E}$ )

Blaualgen stellen an den meisten Extremstandorten der Welt Pioniere der Vegetation dar. Das zeigte erneut die Untersuchung der Algen Aldabras, wo etwa 110 Cyanophyten-Taxa festgestellt wurden, während alle anderen Algengruppen zusammen mit 55 Taxa vertreten waren. Nach der Bestandesaufnahme wurden besonders die ökologischen Bedingungen, unter denen einzelne Arten gehäuft auftreten, bearbeitet.

Diskussion. Sie beschäftigte sich hauptsächlich mit methodischen Fragen, wobei der Einsatz von Lochkarten durch den Autor besonderes Interesse fand.

Le Campron-Alsumard, T.: Cyanophycées marines endolithes colonisant les surfaces rocheuses dénudées (Etages Supralittoral et Médiolittoral de la région de Marseille) (Seite 552)

Es wurden endolithische Blaualgen untersucht, welche Felsen des Supra- und Mediolitorals in der Gegend von Marseille besiedeln. Intensiv wurde Entophysalis deusta studiert: Im Supralitoral ist sie eher epilithisch, dringt nur wenig in den Felsen ein. Im stärker benetzten Mediolitoral bildet sie endolithische, fädige Stadien aus.

Golubic, S.: Taxonomic Interpretation of Marine Endolithic Cyanophyta

Der Kreis der ökologisch extrem spezialisierten mikroskopischen Algen, welche in ihre Kalksubstrate eindringen, umfasst Formen verschiedener systematischer Stellung. Die meisten sind Blaualgen, deren taxonomische Stellung im einzelnen noch unklar und über die auch sonst noch wenig bekannt ist. Es wurden neue Methoden entwickelt, um die Algen von ihrer harten Kalkumgebung zu isolieren, ohne ihre ursprüngliche Form und Wachstumsorientierung zu zerstören. Mit einer Kunstharzausgussmethode konnten Bohrmuster erhalten werden, welche neue charakteristische Merkmale in Erscheinung treten liessen, die nützliche Hinweise für die taxonomische Einstufung der bohrenden Formen lieferten. Taxa aus den folgenden Gattungen wurden studiert und miteinander verglichen: Hormathonema, Hyella, Solentia, Brachytrichia, Mastigocoleus, Plectonema.

Diskussion. Das Hauptinteresse galt der beeindruckenden Untersuchungsmethode Golubičs. Man wünschte, mit der gleichen Methode möchten Probleme um endolithische Süsswasserformen angegangen werden. 
EICHenberger, E.: Sauerstoffhaushalt einer blaualgenhaltigen Biozönose in Modellfliessgewässern (Die Arbeit wird in erweiterter Form später publiziert)

Die für die Sauerstoffbilanz in der untersuchten Biozönose wichtigen Prozesse sind: Die Photosynthese als Sauerstoff produzierender Prozess, heterotrophe Organosynthese, die Zellatmung der lebenden Biomasse und die Abbauprozesse abgestorbener Organismen als Sauerstoff konsumierende Prozesse.

\section{Exkursionen}

Zwei eintägige Exkursionen - die eine ins Berner Oberland, die andere auf die Rigi - boten Gelegenheit zum Studium der Blaualgen in der Spritzzone von Wasserfällen (Reichenbachfall, Staubbachfall), auf überrieselten Felsen und in Tintenstrichen. Sie gaben den Teilnehmern zudem einen Einblick in die Bergwelt der Schweizer Alpen.

\section{Rückblick und Ausblick}

Das 5. Symposium der Internationalen Arbeitsgemeinschaft für Cyanophytenforschung über Fragen der Blaualgensystematik wies erfreulicherweise einen grösseren Teilnehmerkreis auf als die vier ersten Symposien, fanden sich doch zum erstenmal auch Kollegen aus aussereuropäischen Gebieten (Indien, USA) ein. Grundsätzlich waren sich auch diesmal die Teilnehmer einig: Die Blaualgentaxonomie kann nur entscheidend gefördert werden, indem systematische Einheiten sorgfältig revidiert werden. Das verlangt gründliches, oft langwieriges Studium der zu bearbeitenden Formenkreise. Morphologische und ökologische Untersuchungen an Naturmaterial bilden die Basis. Parallel dazu sind experimentelle Untersuchungen mit Klonkulturen genau definierter Herkunft oft unerlässlich: In vielen Fällen gestatten nur sie, zu entscheiden, ob ein bestimmtes Merkmal genetisch fixiert oder ökotypisch ist. Diese Grundsätze werden auch für die Themengestaltung und die Arbeitsweise an unseren nächsten Zusammenkünften richtungweisend sein.

Adresse des Autors:

Dr. A. Zehnder, Tannenhofstrasse 5, 5432 Neuenhof (Schweiz).

Separatdruck aus der Schweiz. Zeitschrift für Hydrologie 33, Fasc. 2 (1970) Herausgegeben mit Unterstützung der Stiftung der Wirtschaft zur Förderung des Gewässerschutzes in der Schweiz 